\title{
Design and calibration of a high-sensitivity and high-accuracy polarimeter based on liquid crystal variable retarders *
}

\author{
Jing Guo ${ }^{1,2}$, De-Qing Ren ${ }^{3,1,2}$, Cheng-Chao Liu ${ }^{1,2}$, Yong-Tian Zhu ${ }^{1,2}$, Jiang-Pei Dou ${ }^{1,2}$, Xi \\ Zhang ${ }^{1,2}$ and C. Beck ${ }^{4}$ \\ ${ }^{1}$ National Astronomical Observatories/Nanjing Institute of Astronomical Optics \& Technology, \\ Chinese Academy of Sciences, Nanjing 210042, China; jguo@niaot.ac.cn \\ ${ }^{2}$ Key Laboratory of Astronomical Optics \& Technology, Nanjing Institute of Astronomical Optics \& \\ Technology, Chinese Academy of Sciences, Nanjing 210042, China \\ 3 Physics \& Astronomy Department, California State University Northridge, 18111 Nordhoff Street, \\ Northridge, California 91330-8268, USA \\ ${ }^{4}$ National Solar Observatory, USA, Boulder, CO 80303
}

Received 2016 August 12; accepted 2016 October 13

\begin{abstract}
Polarimetry plays an important role in the measurement of solar magnetic fields. We developed a high-sensitivity and high-accuracy polarimeter (HHP) based on nematic liquid crystal variable retarders (LCVRs), which has a compact setup and no mechanical moving parts. The system design and calibration methods are discussed in detail. The azimuth error of the transmission axis of the polarizer as well as the fast axes of the two LCVRs and the quarter-wave plate were determined using dedicated procedures. Linearly and circularly polarized light were employed to evaluate the performance of the HHP. The experimental results indicate that a polarimetric sensitivity of better than $5.7 \times 10^{-3}$ can be achieved by using a single short-exposure image, while an accuracy on the order of $10^{-5}$ can be reached by using a large number of short-exposure images. This makes the HHP a high-performance system to be used with ground-based solar telescope for high-precision solar magnetic field investigations.
\end{abstract}

Key words: instrumentation: polarimeters — methods: laboratory — Sun: magnetic fields — techniques: polarimetric

\section{INTRODUCTION}

It is well-known that activity on the Sun is dominated by its magnetic fields. As such, polarized radiation from the Sun contains a wealth of information on its magnetic fields, and solar polarimetry provides us a great opportunity to research and interpret solar phenomena related to the spatial structure of local solar magnetic fields, such as solar wind, radio wave flux, solar flares, coronal mass ejections, coronal heating and sunspots (del Toro Iniesta 2003). The aim of solar polarimetry is to determine the full Stokes parameters of the incident light, and usually together with a spectrograph the information of solar magnetic fields can be obtained through Zeeman or Hanle effect (Stenflo 2013). As early in 1908, Hale used a Fresnel rhomb and a rotatable Nicol prism (i.e., polarimetry) to photographically detect the Zeeman splitting of spectral lines observed in sunspots and to validate the presence of strong magnetic

* Supported by the National Natural Science Foundation of China. 
fields in sunspots (Hale 1908). From that beginning, polarimetry has played an important role in solar observations, and a polarimeter is considered to be an inherent part of solar instrumentation (Elmore 2011).

The 1-meter New Vacuum Solar Telescope (NVST) is a new-generation large high-technology solar facility of China (Liu \& Xu 2011; Wang et al. 2013). Its scientific goals include observing the Sun with very high spatial and spectral resolution in the wavelength range from 0.3 to $2.5 \mu \mathrm{m}$, detecting small-scale structures and fine details in the evolution of the solar magnetic fields, and studying other key questions related to solar activities (Liu 2014). Currently, the polarimeter of the NVST employs a rotating modulation system with classical wave plates (Liu et al. 2014). This kind of traditional polarization modulator is or was also used by many other solar polarimeters, e.g., the Advanced Stokes Polarimeter (ASP; Skumanich et al. 1997), the Diffraction-Limited Spectro-Polarimeter (DLSP; Sankarasubramanian et al. 2003), the Polarimetric Littrow Spectrograph (POLIS; Beck et al. 2005), and the Spectro-Polarimeter for Infrared and Optical Regions (SPINOR; Socas-Navarro et al. 2006), etc. However, the utilization of rotating parts can lead to instrument jitter that decreases measurement accuracy and increases mass and volume of the system as well (Iglesias et al. 2016). These disadvantages are more serious for space-borne instruments where the resources are very limited and have the risk of a mechanical failure (Álvarez-Herrero et al. 2011).

Fortunately, with the development of liquid crystals (LC), liquid crystal variable retarders (LCVRs), which function as variable wave plates, are an attractive alternative to traditional rotary polarizing optics, since the polarization state of the light beam can be actively controlled by varying the voltage applied to the LCVR without any mechanical movements (Shih et al. 2014; Zangrilli et al. 2003). The absence of moving parts can provide quick switching times on the order of a few milliseconds. Together with short exposure times, this makes it possible to freeze atmospheric turbulence with a ground-telescope. Furthermore, LCVRs have a broad wavelength range in the visible and low optical losses. As a consequence, polarization modulation by LCVRs is very promising, and several LCVR-based solar polarimeters have been developed in recent years, e.g., the Facility InfraRed Spectropolarimeter (FIRS) installed at the Dunn Solar Telescope (DST) (Jaeggli et al. 2010), the KIS/IAA Visible Imaging Polarimeter (VIP) operated at the German Vacuum Tower Telescope (Beck et al. 2010), and the Imaging Magnetograph eXperiment (IMaX) on board the Sunrise balloon-borne solar observatory (Martínez Pillet et al. 2011). This type of polarimeter is able to reach a high polarimetric sensitivity on the order of $10^{-3}$.

In this paper, we describe the development and testing of a high-sensitivity and high-accuracy polarimeter (HHP), which consists of a pair of temperature-stabilized nematic LCVRs, a Wollaston prism (WP) and a charge-coupled device (CCD) camera. The HHP is superior to the above LCVR-based polarimeters in polarimetric accuracy and system speed. Section 2 provides a detailed description of the system design and associated algorithms developed to achieve the design goal. Section 3 gives our calibration procedures for the polarization elements. Section 4 describes polarimetric results and how the polarimetric sensitivity and accuracy were evaluated. Section 5 presents the discussion. Finally, we present our conclusions in Sec. 6.

\section{THEORY}

For ground-based solar polarimetry, one should particularly pay attention to the atmospheric seeing, since it will introduce spurious polarization signals. With most of the seeing power contained in the 1-100 Hz frequency range (Judge et al. 2004), a modulation frequency of the order of $100 \mathrm{~Hz}$ is required to reliably reconstruct the four polarization components $I, Q, U$ and $V$ (Krishnappa \& Feller 2012). Consequently, seeing-induced crosstalk can be eliminated by using a very high modulation frequency in a single-beam polarimeter. For instance, the Zürich Imaging Polarimeter (ZIMPOL) employs piezoelastic modulators with a frequency of $50 \mathrm{kHz}$, but a dedicated CCD detector must be used, in which three out of four rows of the sensor are covered and a micro-lenses array is attached (Gandorfer et al. 2004). Although our LCVRs can finish the polarization modulation scheme within $1.2 \mathrm{~ms}$ (totally four independent retardance combinations) by using an appropriate strategy (retardance decrease takes much shorter than the equivalent retardance increase), the modulation frequency is mainly limited by the 
frame rate of the camera we currently use (109 fps), which will be updated very soon with a fast camera with a frame rate of up to 400-800 fps. Currently, our design uses a dual-beam configuration and a Wollaston prism which encodes polarization into intensity with opposite signs for the two beams. The seeing-induced crosstalk thus cancels out during the demodulation procedures.

\subsection{System Design}

In the laboratory, the light feed optics was composed of a He-Ne laser $(\lambda=632.8 \mathrm{~nm})$, a collimating lens, a pinhole ( $\mathrm{PH})$, and a field stop (FS). A nanoparticle linear polarizer (P, extinction ratio $>10^{5}: 1$ in the range 600-1200 nm) plus a quarter-wave plate (QWP, @632.8 nm) acted as polarization states generator (PSG), which was used to generate different known polarization states that were needed to evaluate the polarimetric accuracy of the system. A pair of LCVRs are employed as polarization modulator and a Wollaston prism as dual-beam polarization analyzer. The fast axes of the LCVR1 and LCVR2 are mounted at $0^{\circ}$ and $45^{\circ}$, respectively, and one transmission axis of the Wollaston prism is oriented at $0^{\circ}$. The LCVRs (manufactured by Thorlabs) are AR coated for visible light from 350-700 $\mathrm{nm}$. They can generate a wide retardance range from $30 \mathrm{~nm}$ to $\lambda$. The LC controller LCC25 provides active DC offset compensation while applying an AC voltage. It is worth noting that the properties of the LC materials are temperature-dependent, and the generated retardance will deviate from its desired value owing to the temperature variation (Heredero et al. 2007). Therefore, each LCVR is equipped with a temperature sensor and heater, which will hold the temperature of the retarder constant to within $\pm 0.1^{\circ} \mathrm{C}$ when used with a TC200 temperature controller. The temperature stabilization provides constant retardance even if the ambient temperature changes and also allows for faster switching times. The Wollaston prism (manufactured by Altechnais) is made of quartz (extinction ratio $>2 \times 10^{3}: 1$ in the range 200-2300 $\mathrm{nm}$ ) with an angular field of $1^{\circ} @ 632.8 \mathrm{~nm}$. A non-polarizing beamsplitter (BS) in front of the CCD camera and a high accuracy optical power meter (OPM, with a resolution of $1 \mathrm{nW})$ were used in the azimuth calibrations of the polarization elements (see Sec. 3). The CCD (manufactured by Imperx, model: B0610, readout noise: 16 electrons) is a 16-bit camera with $640 \times 480$ pixels and a pixel size of $7.4 \mu \mathrm{m} \times 7.4 \mu \mathrm{m}$. In the experiments, the LCVRs and the CCD camera were controlled by LabVIEW, and working temperatures of the LCVRs were set to $30.0^{\circ} \mathrm{C}$. The optical layout of the HHP is shown in Fig. 1.

\subsection{Modulation and Demodulation Algorithms}

According to the HHP setup and Mueller calculus, the relationship between input and output Stokes vectors $S_{\text {in }}$ and $S_{\text {out }}$ is given by

$$
S_{\text {out }}=M_{W} \cdot M_{L C V R 2} \cdot M_{L C V R 1} \cdot S_{\text {in }}
$$

where $M_{L C V R}$ and $M_{W}$ are Mueller matrices of the LCVR and the Wollaston prism, respectively, which can be expressed by

$$
\begin{gathered}
M_{L C V R i}=\left(\begin{array}{cccc}
1 & 0 & 0 & 0 \\
0 & \cos ^{2} 2 \alpha_{i}+\sin ^{2} 2 \alpha_{i} \cos \delta_{i} & \cos 2 \alpha_{i} \sin 2 \alpha_{i}\left(1-\cos \delta_{i}\right) & -\sin 2 \alpha_{i} \sin \delta_{i} \\
0 & \cos 2 \alpha_{i} \sin 2 \alpha_{i}\left(1-\cos \delta_{i}\right) & \sin { }^{2} 2 \alpha_{i}+\cos ^{2} 2 \alpha_{i} \cos \delta_{i} & \cos 2 \alpha_{i} \sin \delta_{i} \\
0 & \sin 2 \alpha_{i} \sin \delta_{i} & -\cos 2 \alpha_{i} \sin \delta_{i} & \cos \delta_{i}
\end{array}\right) \\
M_{W}=\frac{1}{2}\left(\begin{array}{cccc}
1 & \cos 2 \beta^{ \pm} & \sin 2 \beta^{ \pm} & 0 \\
\cos 2 \beta^{ \pm} & \cos 2 \beta^{ \pm} & \cos 2 \beta^{ \pm} \sin 2 \beta^{ \pm} & 0 \\
\sin 2 \beta^{ \pm} & \cos 2 \beta^{ \pm} \sin 2 \beta^{ \pm} & \sin ^{2} 2 \beta^{ \pm} & 0 \\
0 & 0 & 0 & 0
\end{array}\right)
\end{gathered}
$$

where $\alpha_{i}$ and $\delta_{i}$ denote the azimuth of the fast axis and the retardance of the $i$-th LCVR, respectively, $\beta$ is the azimuth of the transmission axis of the Wollaston prism, and the positive and negative signs indicate two orthogonal polarized parts, respectively. 


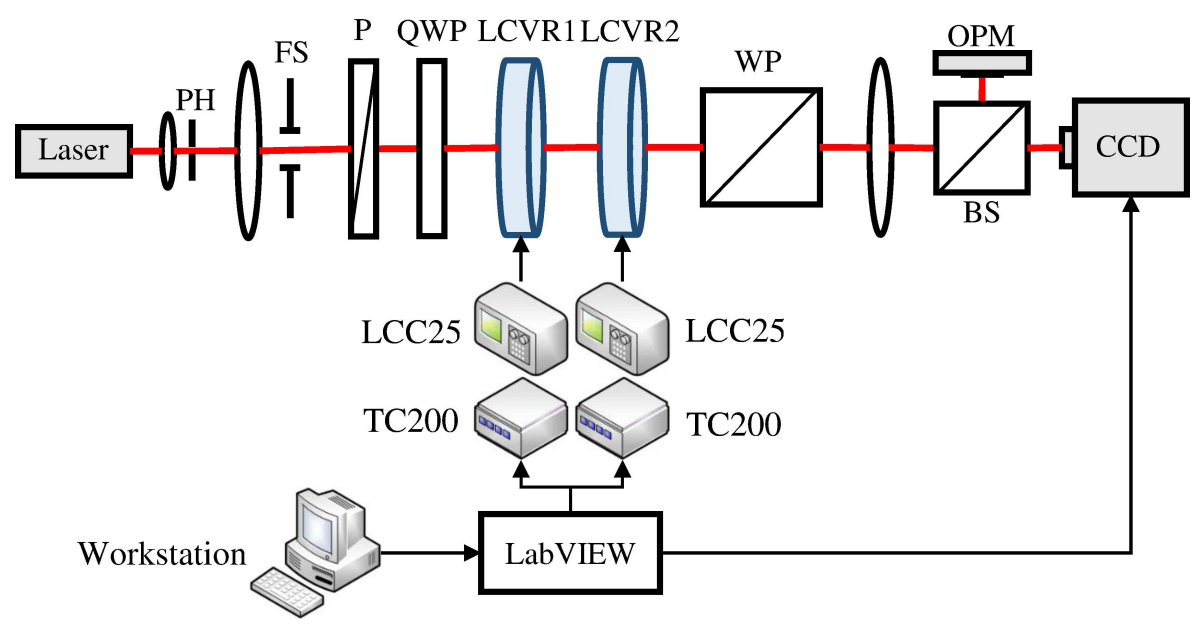

Fig. 1 Optical layout of the HHP. PH: pinhole. FS: field stop. P: polarizer. QWP: quarter-wave plate. LCVR: liquid crystal variable retarders. WP: Wollaston prism. LCC25: LC controller. TC200: temperature controller. BS: beamsplitter. OPM: optical power meter. CCD: chargecoupled device.

The intensity measured by the CCD camera is given by

$$
L^{ \pm}=\frac{1}{2}\left(I \pm \cos \delta_{2} Q \pm \sin \delta_{1} \sin \delta_{2} U \mp \cos \delta_{1} \sin \delta_{2} V\right)
$$

where $L^{+}$and $L^{-}$are the intensities of the two orthogonal polarized images, respectively. In order to retrieve the four Stokes parameters in Eq. (4), at least four different modulation states have to be constructed. Consequently, a modulation strategy from the Imaging Magnetograph eXperiment (IMaX) is used, since it makes polarization modulation efficiencies attain their maximum values, while guaranteeing fast switching times of the LCVRs (Uribe-Patarroyo et al. 2008). The retardance combinations are as follows

$$
\left(\delta_{1}, \delta_{2}\right)=\left(315^{\circ}, 305.264^{\circ}\right),\left(315^{\circ}, 54.736^{\circ}\right),\left(225^{\circ}, 125.264^{\circ}\right),\left(225^{\circ}, 234.736^{\circ}\right)
$$

Finally, the four Stokes parameters can be computed using the observed intensities of the two orthogonally polarized images for the polarization states described above by

$$
\left\{\begin{array}{l}
I=\frac{1}{4}\left[\left(L_{1}^{+}+L_{2}^{+}+L_{3}^{+}+L_{4}^{+}\right)+\left(L_{1}^{-}+L_{2}^{-}+L_{3}^{-}+L_{4}^{-}\right)\right] \\
Q=\frac{\sqrt{3}}{4}\left[\left(L_{1}^{+}+L_{2}^{+}-L_{3}^{+}-L_{4}^{+}\right)-\left(L_{1}^{-}+L_{2}^{-}-L_{3}^{-}-L_{4}^{-}\right)\right] \\
U=\frac{\sqrt{3}}{4}\left[\left(L_{1}^{+}-L_{2}^{+}-L_{3}^{+}+L_{4}^{+}\right)-\left(L_{1}^{-}-L_{2}^{-}-L_{3}^{-}+L_{4}^{-}\right)\right] \\
V=\frac{\sqrt{3}}{4}\left[\left(L_{1}^{+}-L_{2}^{+}+L_{3}^{+}-L_{4}^{+}\right)-\left(L_{1}^{-}-L_{2}^{-}+L_{3}^{-}-L_{4}^{-}\right)\right] .
\end{array}\right.
$$

where $L_{i}$ is the measured intensity under the $i$-th retardance combination of the LCVRs.

\section{CALIBRATIONS}

Although the two LCVRs, the Wollaston prism, the polarizer and the quarter-wave plate are mounted on separate rotation stages, it is essential to calibrate the azimuths of these polarization elements first. Their actual azimuths may differ from the ideal values owing to the mounting errors. Any misalignment is a major factor that limits the polarimetric accuracy. To address this issue, a reference horizontal direction along the long side of focal plane of the CCD camera is selected and all azimuths are calibrated with respect to the reference direction in the following sequence. 


\subsection{Azimuth of Transmission Axis of the Wollaston Prism}

After the light feed optics have been set up, the imaging optics and the CCD camera are installed, the Wollaston prism is mounted and the two light spots created by the Wollaston are adjusted to be located in the left and right half of the focal plane. Then, we can calibrate one azimuth of the transmission axes by rotating the Wollaston prism while calculating central coordinates of the two light spots. When their longitudinal coordinates are equal to each other, the orientation of the transmission axis is parallel to the reference direction, and the Wollaston prism is fixed in that orientation. A fast and effective centroid algorithm is employed

$$
\left\{\begin{array}{c}
x_{c e n t}^{ \pm}=\frac{\sum_{i=1}^{M} \sum_{j=1}^{N} j L_{i, j}^{ \pm}}{\sum_{i=1}^{M} \sum_{j=1}^{N} L_{i, j}^{ \pm}} \\
y_{\text {cent }}^{ \pm}=\frac{\sum_{i=1}^{M} \sum_{j=1}^{N} i L_{i, j}^{ \pm}}{\sum_{i=1}^{M} \sum_{j=1}^{N} L_{i, j}^{ \pm}}
\end{array}\right.
$$

where $M$ and $N$ are pixel numbers of length and width of the focal plane, respectively, for the positive sign, $i=1 \sim M / 2$; for the negative sign, $i=M / 2 \sim M$.

Fig 2 illustrates the calibrated azimuth calculated using the central coordinates of the two light spots in 100 measurements. The result reveals that one azimuth of the Wollaston prism is within $1^{\prime}$ with respect to the reference horizontal direction after calibration.

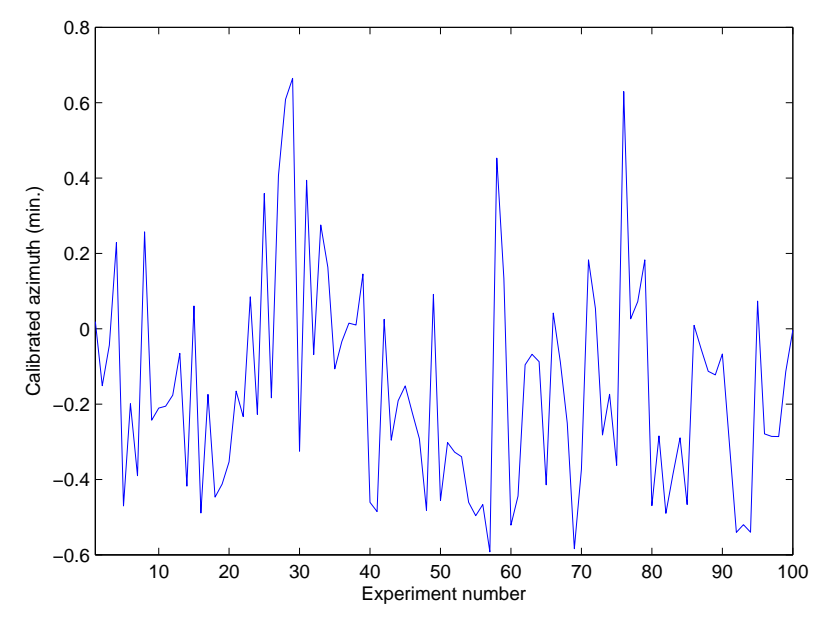

Fig. 2 Calibrated azimuth of transmission axis of the Wollaston prism with respect to the reference horizontal direction.

\subsection{Azimuth of Transmission Axis of the Polarizer}

The polarizer is then added into the optical path, and according to the Malus law, one of light spots will reach the minimum intensity when the azimuth of the transmission axis of the polarizer is parallel to the reference direction (perpendicular to the other transmission axis of the Wollaston prism). In the experiments, we found that the intensity has a flat area within a small azimuth range around the crossed orientation, where all intensities are about equal to the minimum value. Therefore, the high accuracy optical power meter had to be used. We first adjusted the intensity to some specific values and recorded their azimuths, and then the parallel azimuth can be determined by calculating the mean value of the recorded azimuths. A schematic diagram of the calibration method is shown in Fig. 3, while the results are listed in Table 1. 


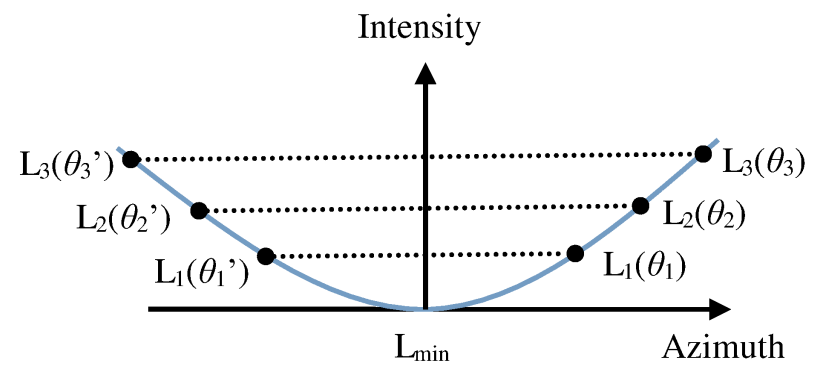

Fig. 3 Schematic diagram of the calibration method of transmission axis of the polarizer.

Table 1 Azimuth calibration of transmission axis of the polarizer.

\begin{tabular}{cccc}
\hline Intensity $\left(10^{-2} \mu W\right)$ & Recorded azimuth & Mean value & Calibrated azimuth \\
\hline 4.0 & $1^{\circ} 30^{\prime} ;-2^{\circ} 40^{\prime}$ & $-35^{\prime}$ & \\
5.0 & $2^{\circ} 30^{\prime} ;-4^{\circ} 0^{\prime}$ & $-45^{\prime}$ & $-43^{\prime}$ \\
6.0 & $3^{\circ} 20^{\prime} ;-5^{\circ} 0^{\prime}$ & $-50^{\prime}$ & \\
\hline
\end{tabular}

Table 2 Azimuth calibration of fast axes of the LCVRs and the QWP.

\begin{tabular}{ccccc}
\hline Elements & Intensity $\left(10^{-2} \mu W\right)$ & Recorded azimuth & Mean value & Calibrated azimuth \\
\hline \multirow{3}{*}{ LCVR1 } & 4.0 & $2^{\circ} 20^{\prime} ;-2^{\circ} 0^{\prime}$ & $10^{\prime}$ & \\
& 5.0 & $3^{\circ} 10^{\prime} ;-3^{\circ} 15^{\prime}$ & $-3^{\prime}$ & $3^{\prime}$ \\
& 6.0 & $4^{\circ} 10^{\prime} ;-4^{\circ} 5^{\prime}$ & $3^{\prime}$ & \\
LCVR2 & 4.0 & $0^{\circ} 40^{\prime} ;-2^{\circ} 10^{\prime}$ & $-45^{\prime}$ & \\
& 5.0 & $1^{\circ} 50^{\prime} ;-3^{\circ} 0^{\prime}$ & $-35^{\prime}$ & $38^{\prime}$ \\
QWP & 6.0 & $2^{\circ} 30^{\prime} ;-3^{\circ} 40^{\prime}$ & $-35^{\prime}$ & \\
& 4.0 & $2^{\circ} 0^{\prime} ;-1^{\circ} 55^{\prime}$ & $3^{\prime}$ & \multirow{2}{*}{} \\
& 5.0 & $2^{\circ} 30^{\prime} ;-2^{\circ} 20^{\prime}$ & $5^{\prime}$ & \\
\hline
\end{tabular}

\subsection{Azimuths of Fast Axes of the LCVRs and the Quarter-Wave Plate}

When the retarder is placed between the polarizer and the Wollaston prism, the transmitted intensity is given by

$$
L=L_{0}\left[\cos ^{2} \alpha-\sin 2 \varphi \sin 2(\varphi-\alpha) \sin ^{2}(\delta / 2)\right]
$$

where $\alpha$ is the relative azimuth of the transmission axis between the polarizer and the Wollaston prism, $\varphi$ is the relative azimuth of the fast axis between the retarder and the transmission axis of the polarizer, and $\delta$ is the retardance. If $\alpha=90^{\circ}$ and $\varphi=0$, then the azimuth of the fast axis of the retarder is parallel to the reference direction and the transmitted intensity will reach the minimum value. The azimuths of the fast axes of the retarders are calibrated separately in a manner similar to that presented in Sec. 3.2. Calibrated results are shown in Table 2.

\subsection{Retardances of the LCVRs}

Since the LCVRs have a variable retardance, it is important to obtain the relationship between applied voltages and generated retardances. According to setup of the HHP, a voltage-dependent transmission technique is applied, and the retardance can be calculated from the transmitted intensities ratio $L_{\perp} / L_{\|}$ (Wu et al. 1984; Liu et al. 2016). The merit of this method is that effect of intensity fluctuation of the 
Table 3 Four voltages of each LCVR determined after retardance calibration.

\begin{tabular}{ccccccccc}
\hline & \multicolumn{3}{c}{ LCVR1 } & \multicolumn{7}{c}{ LCVR2 } \\
\hline Desired retardance (deg.) & 315 & 315 & 225 & 225 & 305.264 & 54.736 & 125.264 & 234.736 \\
Applied voltage (V) & 1.164 & 1.164 & 1.578 & 1.578 & 1.280 & 4.617 & 2.480 & 1.614 \\
\hline
\end{tabular}

light source can be eliminated. One LCVR is placed between the polarizer and the Wollaston prism. The transmission axis of the polarizer is at $0^{\circ}$ and the fast axis of the LCVR is at $45^{\circ}$ as shown in Fig. 4.

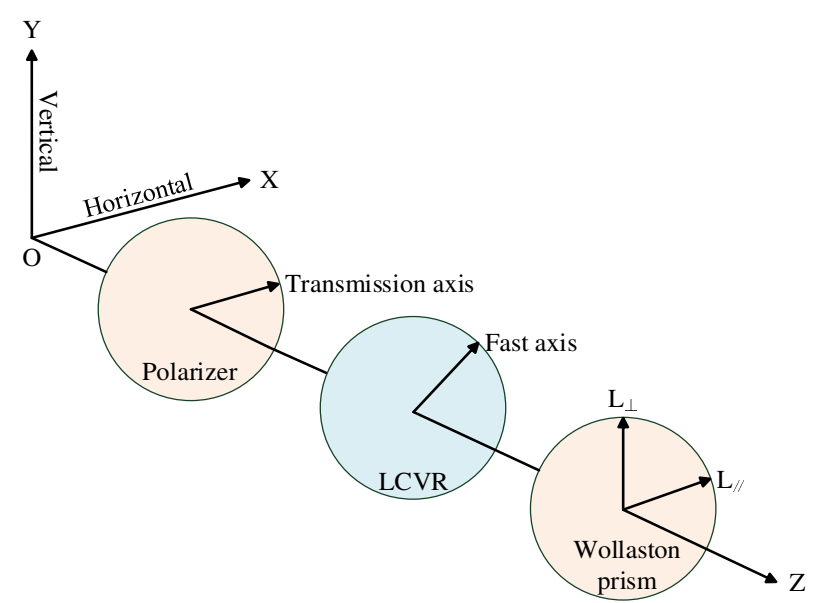

Fig. 4 Configuration of retardance calibration of the LCVR.

The two LCVRs are calibrated individually by applying voltages from 0 to $7 \mathrm{~V}$ with an interval of $0.01 \mathrm{~V}$, and then the retardance are interpolated with an interval of $0.001 \mathrm{~V}$. The results are shown in Fig. 5.

Fig. 5 indicates that relationship between applied voltages and generated retardances apparently exhibits non-linearity. The retardance keeps at a constant value when the voltage is less than a threshold of about $1 \mathrm{~V}$. When the voltage is increased beyond the threshold, the retardance decreases dramatically in a certain voltage range at first and then gradually converges to a nonzero value. This is due to the LC molecules that are anchored at the substrate because they cannot be tilted by the electric field.

We developed an automatically calibration procedure by LabVIEW, which is convenient and fast. The calibration for each LCVR can be accomplished within 3 minutes. The four voltages of each LCVR that need to be applied to generate the desired retardances are shown in Table 3.

\section{RESULTS}

In the experiments, all acquired images have been processed with dark subtraction and flat field at first. A typical results of retrieved Stokes parameters from a linearly horizontal polarized light $(1,1,0,0)^{T}$ are shown in Fig. 6. According to the diameter of the light spot, we select a plot area of $25 \times 25$ pixels. Stokes $Q, U$, and $V$ are normalized to the intensity $I$.

Fig. 6 reveals that the dual-beam analysis successfully retrieved the four Stokes parameters and that the intensity $I$ shows a typical distribution of a light spot. The values of Stokes $Q / I, U / I$ and $V / I$ agree well with that of a linear horizontal polarized light. We found that the retrieved results began to depart from their theoretical values when the area is larger than $18 \times 18$ pixels, since their signal-to-noise ratio $(\mathrm{S} / \mathrm{N})$ is lower than $\sim 10^{2}$. Therefore, the performance of the HHP is evaluated using polarimetric results 

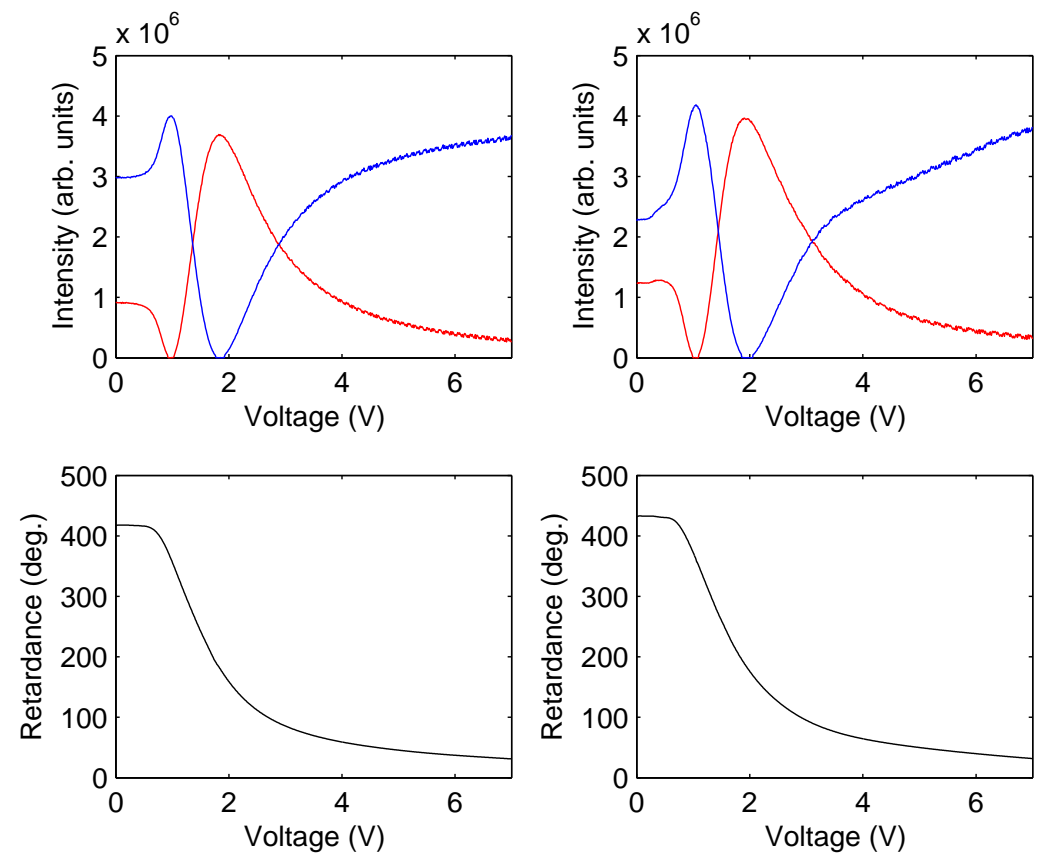

Fig. 5 Retardance calibration. left: LCVR1 and right: LCVR2. red line: $L_{\perp}$ and blue line: $L_{\|}$.

Table 4 Polarimetric sensitivity evaluated by different polarization states.

\begin{tabular}{ccccccc}
\hline & \multicolumn{5}{c}{ Polarization states } \\
LP0 & LP45 & LP90 & LP135 & RCP & LCP \\
\hline RMS noise & $3.7 \times 10^{-3}$ & $4.0 \times 10^{-3}$ & $4.1 \times 10^{-3}$ & $3.5 \times 10^{-3}$ & $5.1 \times 10^{-3}$ & $5.7 \times 10^{-3}$ \\
\hline
\end{tabular}

within this area. The polarimetric sensitivity is measured directly from one set of images that consists of 4 images as discussed previously according to the previous equations, which yields $4.2 \times 10^{-3}$ in our measurement. The accuracy is typically quantified by the root-mean-square (RMS) noise of the Stokes parameters based on the sensitivity measurements in a large number of sets of measurements (Beck et al. 2005). Since this RMS value decreases with the square root of the images number, as shown in Fig. 7, a 10,000 sets of image, which is a typical value for the accuracy evaluation (Povel 1995),will yield an accuracy of $4.6 \times 10^{-5}$ for our polarimeter system.

Furthermore, four linearly (polarizer at $0^{\circ}, 45^{\circ}, 90^{\circ}$, and $135^{\circ}$, respectively) and two circularly (right-handed and left-handed) polarization states are generated by the PSG, and their separate RMS noises (mean value of 20 measurements per condition) are shown in Table 4.

Table 4 shows that RMS noises are better than $4.1 \times 10^{-3}$ and $5.7 \times 10^{-3}$ for the linearly and circularly polarization states, respectively. Because of retardance error of the quarter-wave plate $(<$ $\lambda / 300$ provided by the manufacturer), polarimetric sensitivity of the circularly polarized light is a little lower than that of the linearly ones. From above analyses, we can conclude that the performance of our HHP is satisfactory and stable. 

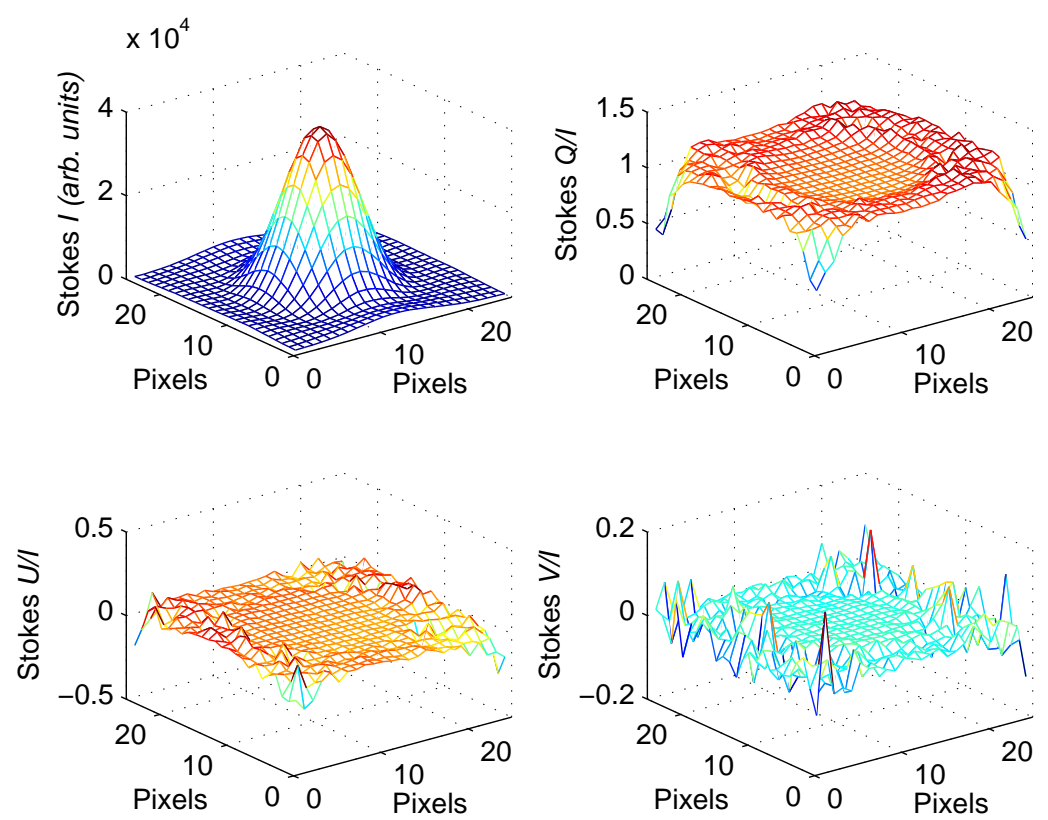

Fig. 6 Retrieved Stokes parameters from a linearly horizontal polarized light.

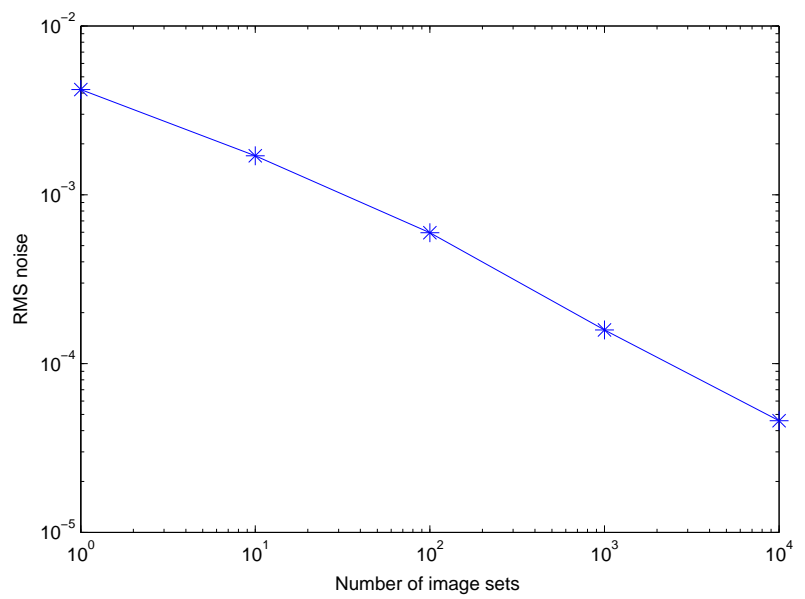

Fig. 7 Measured RMS noise tested by different sets of image.

\section{DISCUSSION}

Under our schedule, a high frame rate (400 fps @ 2048 $\times 512$ pixels and a pixel size of $6.5 \mu \mathrm{m} \times 6.5 \mu \mathrm{m}$ ) and low readout noise (1.4 electrons at highest speed) scientific CMOS camera (manufacture by PCO) is being purchased, which will replace the existing Imperx B0610 camera that has a readout-noise of 16 electrons, and thus will provide a sensitivity better than $5.0 \times 10^{-4}$ and an accuracy of $4.0 \times 10^{-6}$. 
An integral field unit (IFU) that can acquire spectral information across a two-dimensional field of view (i.e. a data cube) in a single exposure is being developed by a team led by Drs. Ren and Beck for the National Solar Observatory (NSO) Horizontal Spectrograph with the DST (Beck et al. 2016). The HHP will be used with the NSO Horizontal Spectrograph for its first test observations in 2017.

As a first step, the HHP (including several dedicated softwares) have been developed and tested in the laboratory, and performance of the HHP were evaluated as well. In the next step, the HHP will be used as a visit instrumentation with the NSO Horizontal Spectrograph for its first test observations in 2017, and at the Yunnan Observatory in a long-term plan. The HHP is also available to the astronomy circles.

Because of the modular design, the HHP can be conveniently interfaced with the telescope and spectrometer, shown in Fig. 1.

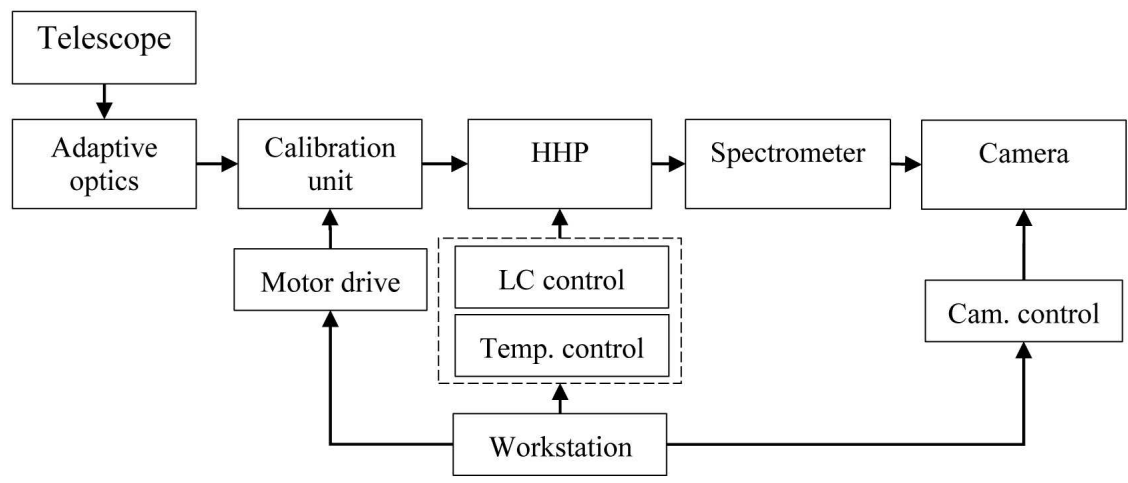

Fig. 8 The HHP interfaced with the telescope and spectrometer.

The light coming from the telescope and the AO system firstly passes through the instrument calibration unit (ICU, which consists of a rotatable linear polarizer and a rotatable quarter-wave plate and is used to derive the response function of the system). The ICU can be inserted or removed from the optical train if necessary. And then, the LCVRs-based modulator of the HHP performs polarization modulation to the incident light and angle of the spectrograph grating can be determined by selecting the desired wavelength. After this, the modulated intensities are received and recorded by the CCD camera. The complete system is controlled by a workstation.

The HHP is initially used to measure the magnetic fields of mid-photosphere using Fe I $6302 \AA$ in the visible. In the future, the HHP will be extended to measure the magnetic fields of photosphere using Si I $10827 \AA$ and high chromosphere using He I $10830 \AA$ in the near-infrared when equipped with an infrared camera.

\section{CONCLUSIONS}

Developing high-performance polarimeters is a persistent goal of solar instrumentation because of their importance for measuring solar magnetic fields. We have developed and described a high-sensitivity and high-accuracy polarimeter based on LCVRs. Its performance is sufficient for using it for accurate polarimetry of solar magnetic fields. We estimate that the updated HHP, with its high-sensitivity and combined with the high temporal resolution of the NSO IFU spectrograph, will be a unique equipment for solar magnetic field observations. In a long-term plan, we will also use our HHP with the NVST existing spectrograph for solar magnetic scientific investigations.

Acknowledgements This work was funded by the National Natural Science Foundation of China (NSFC) (Grant Nos. 11661161011, 11433007, 11220101001, 11328302, 11373005, and 11303064), the 
"Strategic Priority Research Program" of the Chinese Academy of Sciences (Grant No. XDA04075200), the special fund for astronomy of CAS (2015-2016), the special funding for Young Researcher of Nanjing Institute of Astronomical Optics \& Technology, the International Partnership Program of Chinese Academy of Sciences (Grant No.114A32KYSB20160018), as well as the Mt. Cuba Astronomical Foundation.

\section{References}

Álvarez-Herrero, A., Uribe-Patarroyo, N., García Parejo, P., et al. 2011, in Proc.SPIE, Vol. 8160, $81600 \mathrm{Y}$

Beck, C., Bellot Rubio, L. R., Kentischer, T. J., Tritschler, A., \& del Toro Iniesta, J. C. 2010, A\&A, 520, A115

Beck, C., Schmidt, W., Kentischer, T., \& Elmore, D. 2005, A\&A, 437, 1159

Beck, C., Uitenbroek, H., Tritschler, A., et al. 2016, SoPh, in prep.

del Toro Iniesta, J. C. 2003, in Cambridge: Cambridge University Press

Elmore, D. F. 2011, in ASP Conf. Ser., Vol. 437, 309

Gandorfer, A. M., Povel, H. P., Steiner, P., et al. 2004, A\&A, 422, 703

Hale, G. E. 1908, ApJ, 28, 315

Heredero, R. L., Uribe-Patarroyo, N., Belenguer, T., et al. 2007, Appl. Opt., 46, 689

Iglesias, F. A., Feller, A., Nagaraju, K., \& Solanki, S. K. 2016, A\&A, 590, A89

Jaeggli, S. A., Lin, H., Mickey, D. L., et al. 2010, Mem. Soc. Astron. Ital., 81, 763

Judge, P. G., Elmore, D. F., Lites, B. W., Keller, C. U., \& Rimmele, T. 2004, Appl. Opt., 43, 3817

Krishnappa, N., \& Feller, A. 2012, Appl. Opt., 51, 7953

Liu, C.-C., Ren, D.-Q., Zhu, Y.-T., Dou, J.-P., \& Guo, J. 2016, Research in Astron. Astrophys. (RAA), 16,73

Liu, Z. 2014, in ASP Conf. Ser., Vol. 489, 247

Liu, Z., \& Xu, J. 2011, in ASI Conf. Ser., Vol. 2, 9

Liu, Z., Xu, J., Gu, B.-Z., et al. 2014, Research in Astron. Astrophys. (RAA), 14, 705

Martínez Pillet, V., del Toro Iniesta, J. C., Álvarez-Herrero, A., et al. 2011, SoPh, 268, 57

Povel, H. 1995, OptEn, 34, 1870

Sankarasubramanian, K., Elmore, D. F., Lites, B. W., et al. 2003, in Proc.SPIE, Vol. 4843, 414

Shih, W.-T., Hsieh, M.-L., \& Chao, Y.-F. 2014, in Proc.SPIE, Vol. 9200, 920009

Skumanich, A., Lites, B. W., Pillet, V. M., \& Seagraves, P. 1997, AJS, 110, 357

Socas-Navarro, H., Elmore, D., Pietarila, A., et al. 2006, SoPh, 235, 55

Stenflo, J. O. 2013, AARv, 21, 66

Uribe-Patarroyo, U., Álvarez-Herrero, A., Heredero, R. L., et al. 2008, PSSCR, 5, 1041

Wang, R., Xu, Z., Jin, Z.-Y., et al. 2013, Research in Astron. Astrophys. (RAA), 13, 1240

Wu, S.-T., Efron, U., \& Hess, L. D. 1984, Appl. Opt., 23, 3911

Zangrilli, L., Fineschi, S., Loreggia, D., et al. 2003, Mem. Soc. Astron. Ital., 74, 528 